OPEN ACCESS

Edited by:

Kwangcheol Casey Jeong, University of Florida, United States

Reviewed by:

Lin Teng,

Zhejiang University, China Kap-Hoon Han,

Woosuk University, South Korea

${ }^{*}$ Correspondence:

John G. Gibbons jggibbons@umass.edu

Specialty section:

This article was submitted to

Food Microbiology,

a section of the journal

Frontiers in Microbiology

Received: 06 April 2021 Accepted: 22 June 2021

Published: 13 July 2021

Citation:

Chacón-Vargas K, McCarthy CO,

Choi D, Wang L, YU J-H and

Gibbons JG (2021) Comparison of Two Aspergillus oryzae Genomes

From Different Clades Reveals Independent Evolution

of Alpha-Amylase Duplication, Variation in Secondary Metabolism Genes, and Differences in Primary

Metabolism

Front. Microbiol. 12:691296. doi: 10.3389/fmicb.2021.691296

\section{Comparison of Two Aspergillus oryzae Genomes From Different Clades Reveals Independent Evolution of Alpha-Amylase Duplication, Variation in Secondary Metabolism Genes, and Differences in Primary Metabolism}

\author{
Katherine Chacón-Vargas 1,2 , Colin O. McCarthy², Dasol Choij,4, Long Wang ${ }^{5}$, \\ Jae-Hyuk Yu4,6 and John G. Gibbons ${ }^{1,2,7 *}$
}

\begin{abstract}
'Molecular and Cellular Biology Graduate Program, University of Massachusetts, Amherst, MA, United States, ${ }^{2}$ Department of Food Science, University of Massachusetts, Amherst, MA, United States, ${ }^{3}$ Deapertment of Food Science, University of Wisconsin-Madison, Madison, WI, United States, ${ }^{4}$ Department of Bacteriology, and Food Research Institute, University of Wisconsin-Madison, Madison, WI, United States, ${ }^{5}$ State Key Laboratory of Mycology, Institute of Microbiology, Chinese Academy of Sciences, Beijing, China, ${ }^{6}$ Department of Systems Biotechnology, Konkuk University, Seoul, South Korea,

${ }^{7}$ Organismic \& Evolutionary Biology Graduate Program, University of Massachusetts, Amherst, MA, United States
\end{abstract}

Microbes (bacteria, yeasts, molds), in addition to plants and animals, were domesticated for their roles in food preservation, nutrition and flavor. Aspergillus oryzae is a domesticated filamentous fungal species traditionally used during fermentation of Asian foods and beverage, such as sake, soy sauce, and miso. To date, little is known about the extent of genome and phenotypic variation of $A$. oryzae isolates from different clades. Here, we used long-read Oxford Nanopore and short-read Illumina sequencing to produce a highly accurate and contiguous genome assemble of $A$. oryzae 14160, an industrial strain from China. To understand the relationship of this isolate, we performed phylogenetic analysis with $90 \mathrm{~A}$. oryzae isolates and 1 isolate of the $A$. oryzae progenitor, Aspergillus flavus. This analysis showed that $A$. oryzae 14160 is a member of clade $\mathrm{A}$, in comparison to the RIB 40 type strain, which is a member of clade $\mathrm{F}$. To explore genome variation between isolates from distinct $A$. oryzae clades, we compared the $A$. oryzae 14160 genome with the complete RIB 40 genome. Our results provide evidence of independent evolution of the alpha-amylase gene duplication, which is one of the major adaptive mutations resulting from domestication. Synteny analysis revealed that both genomes have three copies of the alpha-amylase gene, but only one copy on chromosome 2 was conserved. While the RIB 40 genome had additional copies of the alpha-amylase gene on chromosomes III, and V, 14160 had a second copy on chromosome II and an third copy on chromosome VI. Additionally, we identified hundreds of lineage specific genes, and putative high impact mutations in genes involved in secondary metabolism, including several of the core biosynthetic 
genes. Finally, to examine the functional effects of genome variation between strains, we measured amylase activity, proteolytic activity, and growth rate on several different substrates. RIB 40 produced significantly higher levels of amylase compared to 14160 when grown on rice and starch. Accordingly, RIB 40 grew faster on rice, while 14160 grew faster on soy. Taken together, our analyses reveal substantial genome and phenotypic variation within $A$. oryzae.

Keywords: Aspergillus oryzae, comparative genomics, Oxford Nanopore sequencing, fermentation, amylase, Koji

\section{INTRODUCTION}

Domestication is an evolutionary process that involves the genetic modification of a species by breeding it in isolation from its ancestral population in an effort to enhance its utility to humans (Larson et al., 2014). Early farmers used selective breeding to continuously cross individuals with desired traits, eventually yielding crops with more food (i.e., larger/more seeds and fruits) that were easier to harvest (i.e., loss of seed shattering in grains), and livestock that were less aggressive and more fertile (Purugganan and Fuller, 2009; Abbo et al., 2014; Larson and Fuller, 2014; Larson et al., 2014). Domestication can also lead to sub specialization and genetic divergence of lineages within a given domesticated species as observed in chickens, which were independently bred for meat and for eggs (Rubin et al., 2010).

In parallel with plants and animals, microbes (bacteria, yeasts, and molds) were also domesticated, most likely because of their role in food preservation (Gibbons and Rinker, 2015; Steensels et al., 2019). Archeological chemistry evidence of ruminant dairy fat from $\sim 8,000$ year old pottery shards in Northern Europe suggest that humans were employing microbial fermentation to produce cheese (Salque et al., 2013). Similarly, the chemical signatures of a "proto rice wine" were discovered embedded in $\sim 9,000$ year old pottery shards from China (McGovern et al., 2004). Further, the long-term relationship between humans and fungi used for food fermentation is evident through the analysis of archeological remains using (i) ancient DNA sequencing (Cavalieri et al., 2003), (ii) microscopy paired with morphological characterization (Liu et al., 2019) and, (iii) direct isolation of characterization of microbial specimens (Aouizerat et al., 2019, 2020).

Saccharomyces yeasts are the most thoroughly studied domesticated fungi. In particular, there are a number of domesticated lineages of Saccharomyces cerevisiae that have been shaped by artificial selection for particular fermentation applications. For example, lineages of beer yeasts have an increased capacity to metabolize maltotriose (a highly abundant sugar in wort) while also producing fewer off-flavor compounds like 4-vinyl guaiacol (Gallone et al., 2016). In addition to yeasts, several filamentous fungi have been domesticated. For instance, the white mold Penicillium camemberti was domesticated for its role in the maturation of soft cheeses (Ropars et al., 2020). Artificial selection in P. camemberti resulted in white color, increased aerial growth, reduced toxin production, and increased inhibition of fungal competitors compared to its progenitor (Ropars et al., 2020). Additionally, Penicillium roqueforti was domesticated for the production of blue cheeses like Roquefort (Dumas et al., 2020). Two distinct lineages of $P$. roqueforti exist that are associated with pre-industrial and industrial cheese production, and possess beneficial traits for these usages.

Aspergillus oryzae is a domesticated filamentous fungal species used in the production of traditionally fermented Asian foods and beverages, such as shoyu, miso, sake, and meju (Machida et al., 2005, 2008; Gibbons et al., 2012; Alshannaq et al., 2018; Watarai et al., 2019). A. oryzae was domesticated from Aspergillus flavus (Geiser et al., 1998; Machida et al., 2005; Gibbons et al., 2012), or perhaps the closely related species Aspergillus aflatoxiformans or Aspergillus minisclerotigenes (Kjaerbolling et al., 2020). As a result of domestication and specialization to the fermented food environment, $A$. oryzae has reduced capacity to produce many secondary metabolites like aflatoxin and cyclopiazonic acid, and increased carbohydrate metabolism, in part due to the duplication of the alpha-amylase encoding gene (Machida et al., 2005; Hunter et al., 2011; Gibbons et al., 2012; Nemoto et al., 2012). Recently, Watarai et al. (2019) sequenced and analyzed the genomes of 82 A. oryzae strains, and identified eight distinct clades. However, little is known about the genome and functional divergence between these A. oryzae groups.

In this study, we used a combination of short-read and longread DNA sequencing to assemble a highly contiguous genome of the clade A isolate A. oryzae 14160, originally isolated from China. To gain insight into $A$. oryzae genome variation, we compared the A. oryzae 14160 genome to the A. oryzae RIB 40 (clade F) reference genome. We also examined phenotypic differences between the two isolates by measuring amylase activity and growth rate on several culture medias. Our results show that $A$. oryzae 14160 and RIB 40 differ substantially in terms of their genomes and phenotypes.

\section{MATERIALS AND METHODS}

\section{Isolates, Fungal Culturing, and DNA Extraction}

A. oryzae 14160 was originally isolated from Xinyang City, Henan Province, China. Spores were cultured in potato dextrose agar (PDA) at $30^{\circ} \mathrm{C}$ for $48 \mathrm{~h}$. DNA was extracted directly from spores following the protocol from Lee et al. (2017). Qubit and Nanodrop were used to quantify DNA. 


\section{Illumina and Oxford Nanopore Sequencing}

PCR-free Illumina libraries were constructed and sequenced by Novogene. Illumina sequencing was conducted in paired-end 150 bp format. Raw reads were deduplicated by Tally using "withquality" and "pair-by-offset" parameters to remove exact pairedend duplicates (Davis et al., 2013). Deduplicated reads were then trimmed with Trim Galore ${ }^{1}$ using "stringency 1," "quality 30," and "length 50" parameters to remove any adaptor sequences and low quality positions. Error correction was performed using the default settings in SPAdes (Bankevich et al., 2012). Data quality was assessed using FASTQC ${ }^{2}$.

Oxford Nanopore (ONT) libraries were prepared using 400 ng of gDNA following the manufacturer's instructions with the 1D Rapid Sequencing Kit (SQK-NSK007). ONT sequencing was performed on a MinION following the manufacturer's instructions. The A. oryzae 14160 library was run for $12 \mathrm{~h}$. ONT reads were mapped against the $A$. oryzae 14160 genome assembly to assess quality of reads using minimap2 (Li, 2018). Raw Illumina and ONT data for A. oryzae 14160 are available through the NCBI SRA under BioProject accession number PRJNA717291.

\section{Phylogenetic Analysis of A. oryzae 14160}

To reconstruct the evolutionary history of $A$. oryzae 14160 we analyzed the phylogenetic relationship of 89 A. oryzae strains from Gibbons et al. (2012) and Watarai et al. (2019) as well as the $A$. oryzae RIB 40 and A. flavus 3357 reference genomes (Machida et al., 2005; Nierman et al., 2015). BioProject accession numbers for samples are as follows: PRJDB7763 for TK-10, TK-11, TK-12, TK-13, TK-14, TK-15, TK-16, TK-17, TK-18, TK-19, TK-1, TK-20, TK-21, TK-22, TK-23, TK-24, TK-25, TK26, TK-27, TK-28, TK-29, TK-2, TK-30, TK-31, TK-32, TK-33, TK-34, TK-35, TK-36, TK-37, TK-38, TK-39, TK-3, TK-40, TK41, TK-42, TK-43, TK-44, TK-45, TK-46, TK-47, TK-48, TK-49, TK-4, TK-50, TK-51, TK-52, TK-53, TK-54, TK-55, TK-56, TK57, TK-58, TK-59, TK-5, TK-60, TK-61, TK-62, TK-63, TK-64, TK-65, TK-66, TK-67, TK-68, TK-69, TK-6, TK-70, TK-71, TK72, TK-73, TK-74, TK-75, TK-76, TK-77, TK-78, TK-79, TK-7, TK-80, TK-81, TK-82, TK-8, and TK-9, and PRJNA164603 for AO_302 (SRRC 302), AO_331 (RIB 331), AO_333 (RIB 333), AO_537 (RIB 537), AO_632 (RIB 632), AO_642 (RIB 642), and AO_949 (RIB 949). First, Illumina whole-genome data was de-duplicated, and adapter and quality trimmed as described above. Next, sequence reads from each isolate were mapped to the A. oryzae RIB40 reference genome with BWA-MEM v0.7.15 (Li and Durbin, 2009, 2010). SAM files were converted into sorted BAM format using the samtools v1.4.1 "view" and "sort" option (Li et al., 2009). Variant calling was performed with GATK using the "Germline short variant discovery" best practices pipeline (McKenna et al., 2010). The GATK "Haplotype Caller" option was used to call SNPs. Genotype calls for the 92 samples were combined using the "GenotypeVCFs" option. SNPs were extracted and filtered using the "SelectVariants"

${ }^{1}$ http://www.bioinformatics.babraham.ac.uk/projects/trim_galore/

${ }^{2}$ https://www.bioinformatics.babraham.ac.uk/projects/fastqc/ and "VariantFiltration" options. SNP filtering was performed using "hard filtering" with the parameters: "QD $<21.0$ $|\mathrm{FS}>0.5||\mathrm{MQ}<60.0| \mid$ MQRankSum $<-0.2 \mid$ | ReadPosRankSum <-4.0 | | SOR > 1.0." Phylogenetic analysis was performed with RAxML (Stamatakis, 2014) with the GTRGAMMA model and 100 bootstrap replicates. The phylogenetic tree was visualized with ggtree (Yu, 2020) and ggplot2 (Wickham, 2009). Phylogenetic analysis was conducted independently with alignments of $243,486,7,641$, and 3,340 SNPs. Phylogenetic trees generated from reduced SNP marker alignments were performed to investigate the impact of linkage on the inferred phylogenetic relationship. SNPs were separated by a minimum of 4 and $10 \mathrm{~Kb}$ for the 7,641, and 3,340 SNP marker sets, respectively.

\section{Genome Assembly and Annotation}

The A. oryzae 14160 genome was assembled using a hybrid approach that combined the short-read Illumina and long-read ONT data. Error correction and genome assembly was performed with the MaSuRCa assembler with the default parameters (Zimin et al., 2013). The quality of the A. oryzae 14160 genome assembly was assessed with QUAST (Gurevich et al., 2013) and genome completeness was evaluated with BUSCO (Simao et al., 2015).

Gene prediction and annotation of A. oryzae 14160 strain were performed using the Funannotate pipeline ${ }^{3}$. Gene model prediction was evaluated with BUSCO (Simao et al., 2015). Functional annotation was performed with Interproscan 5 (Jones et al., 2014) using the default settings and complemented with Phobius (Kall et al., 2004, 2007) for transmembrane topology and signal peptide prediction. Finally, secondary metabolism associated gene clusters were predicted using antiSMASH using the "strict" detection strictness setting (Medema et al., 2011). The A. oryzae 14160 genome assembly is available through the BioProject accession number PRJNA717291.

\section{Whole Genome Alignment}

MUMer was used to align the A. oryzae 14160 assembly to the RIB 40 reference genome using the parameters "-mum," “-b," and "-c" (Delcher et al., 1999). The Nucmer alignment tool was used to identify conserved synteny between the A. oryzae 14160 and RIB 40 genomes using the "-maxmatch" and “-c 1000" parameters. Nucmer output was filtered using the Delta-Filter tool from with the parameter "-I 4000." Alignment coordinates were extracted by the "show-coords" function from MUMmer using the "-r", "c," and "-l" parameters. Whole genome synteny was visualized using Circos (Krzywinski et al., 2009).

\section{Alpha-Amylase Locus Synteny Analysis}

A. oryzae isolates possess between 1 and 4 copies of the alphaamylase encoding gene (Watarai et al., 2019). We compared the alpha-amylase encoding genes and their flanking regions between A. oryzae RIB 40 and A. oryzae 14160 to determine whether the duplication events shared an evolutionary history, or evolved independently. To identify the alpha-amylase encoding genes in A. oryzae 14160 we used BLASTN with the A. oryzae RIB 40

${ }^{3}$ https://github.com/nextgenusfs/funannotate 
amy1 gene (AO090023000944) as the query and A. oryzae 14160 predicted transcripts as the subject, with an $e$-value cutoff of 1e-30 (Altschul et al., 1997). We also conducted this BLASTN search with the $A$. oryzae 14160 genome assembly. These searches yielded three independent loci containing the alpha-amylase encoding gene in the A. oryzae 14160 genome. Finally, the Funannotate annotation was used for validation (each of the three alpha-amylase copies in the A. oryzae 14160 genome were annotated as "AMY1").

Next, we used SimpleSynteny to visualize the synteny between A. oryzae RIB40 and the A. oryzae 14160 alpha-amylase loci, using the default settings (Veltri et al., 2016). To increase confidence that our results were not the byproduct of assembly errors, we also assembled the A. oryzae 14160 genome with Canu (ONT data only) and SPAdes (ONT and Illumina data), using the default settings (Bankevich et al., 2012; Koren et al., 2017). We reasoned that independent misassembles of identical loci would be exceedingly rare. Synteny analysis of each of the three $A$. oryzae 14160 alpha-amylase loci (including five genes upstream and five genes downstream of the alpha-amylase encoding genes) were visualized between the MaSuRCA (primary assembly), Canu, and SPAdes assemblies and the A. oryzae RIB 40 reference genome. Additionally, because some alpha-amylase loci in A. oryzae contain the a transposable element likely responsible for duplications, we used BLASTN to search for the presence of the Tc1/Mariner class putative transposable element (NCBI accession AB072434.1) in the A. oryzae 14160 genome using an $e$-value cutoff of 1e-30. Finally, to provide further evidence for accurate assembly of the alpha-amylase loci, we used BLASTN searches to identify long ONT reads that spanned the alphaamylase gene and flanking genes that differentiated each $A$. oryzae 14160 alpha-amylase loci using an $e$-value cutoff of $1 \mathrm{e}-30$ and a query coverage cutoff of $80 \%$.

\section{Alpha-Amylase Upstream Sequence Analysis}

We aligned the $1 \mathrm{~Kb}$ upstream region of each of the six alphaamylase genes to explore whether divergence between gene copies or strains correlated with amylase activity. Bedtools was used to extract the $1 \mathrm{~Kb}$ upstream region from each alpha-amylase locus (Quinlan and Hall, 2010). The alignment was performed with MAFFT with the following parameters: “-kimura 1," “-op 3.0," and “-ep 0.5” (Katoh and Standley, 2013).

\section{Single Nucleotide Polymorphism Analysis}

We predicted single nucleotide polymorphisms (SNPs) in A. oryzae 14160 vs. the $A$. oryzae RIB 40 reference genome (Machida et al., 2005). A. oryzae 14160 quality and adapter trimmed and error corrected Illumina reads were mapped against the $A$. oryzae RIB 40 reference genome using BWA-MEM v0.7.15 (Li and Durbin, 2009, 2010). SNPs were called using freebayes v1.3.1 with the default settings with the exception of setting ploidy to haploid $(-$ ploidy =1) (Garrison and Marth, 2012). Next, we used vcftools v0.1.14 to filter variants with the following parameters “-remove-indels," “-remove-filtered-all, “-min-meanDP 25," “-minQ 20," “-recode," and “-recode-INFO-all” (Danecek et al., 2011). SNPs from this filtered VCF file were annotated with SnpEff v4.3t using "Aspergillus_oryzae" as the genome database (Cingolani et al., 2012). Using the SnpEff output, we calculated missense variant rate for each gene to identify genes with relatively elevated occurrences of missense variants. Per gene missense variant rate was calculated as:

$$
\text { Missense Variant Rate }=\frac{\text { number of missense variants }}{\text { length of all exons }}
$$

Gene Ontology Enrichment of gene sets with SNP profiles of interest was conducted through the FungiFun 2 server $^{4}$, using the default settings (Priebe et al., 2015).

\section{Identification of Lineage Specific Genes}

To identify gene absences specific to the A. oryzae 14160 and RIB40 genomes, we used control-FREEC to estimate the copy number of each $1 \mathrm{~kb}$ window with a $200 \mathrm{bp}$ step size (Boeva et al., 2012). The following parameters were used: window $=10$, telocentromeric $=0$, minExpectedGC $=0.33$, and maxExpectedGC $=0.63$. To estimate $\mathrm{CNV}$ for each gene, we used a custom perl script that takes gene coordinates and the control-FREEC output as input (CNV_gene-overlap.pl script is available here: https://github.com/DaRinker/PolarBearCNV) (Rinker et al., 2019). Entire genes (a minimum of start codon to stop codon) with copy numbers of zero when mapped against the non-self genome assembly (i.e., A. oryzae RIB 40 vs. $A$. oryzae 14160 reference, or $A$. oryzae 14160 vs. $A$. oryzae RIB 40 reference) were considered lineage specific genes in the reference genome.

\section{Amylase Activity Assays}

We used a quantitative method to measure amylase activity via the Megazyme (Bray, Ireland) alpha-amylase Assay Kit (Ceralpha Method). Short grain sushi rice was sterilized and cooked in distilled water at a ratio of $1: 1.7$ at $121^{\circ} \mathrm{C}$ for $15 \mathrm{~min}$. Fifteen grams of cooked rice was inoculated with $\sim 100,000$ conidia suspended in $20 \mu \mathrm{L} \mathrm{H} \mathrm{H}_{2} \mathrm{O}$ and incubated for $48 \mathrm{~h}$ at $32^{\circ} \mathrm{C}$. The entire sample was transferred into a $50 \mathrm{ml}$ centrifuge tube, washed with $10 \mathrm{ml}$ distilled $\mathrm{H}_{2} \mathrm{O}$ and vortexed for $1 \mathrm{~min}$. A $2 \mathrm{ml}$ aliquot of wash water was transferred to a $5 \mathrm{ml}$ tube and centrifuged at 1,000 RPM for $10 \mathrm{~min}$. A one $\mathrm{mL}$ aliquot of the supernatant was diluted with $49 \mathrm{~mL}$ alpha-amylase buffer. Buffered enzyme extract was preheated at $40^{\circ} \mathrm{C}$ for $5 \mathrm{~min}$ after which a $0.1 \mathrm{~mL}$ aliquot was added to an equal amount of Megazyme Ceralpha Amylase HR Reagent in triplicate and maintained at $40^{\circ} \mathrm{C}$ for $10 \mathrm{~min}$. Next, $1.5 \mathrm{~mL} 1 \%$ sodium triphosphate solution was added to halt the reaction. Control samples were prepared by immediately adding the sodium triphosphate solution to the enzyme-substrate solution. The samples and controls were transferred into a 24 well microplate and absorbance was measured at $405 \mathrm{~nm}$.

We also used an iodine-based qualitative assay to examine amylase activity (Fuwa, 1954) while isolates grew on starch agar

\footnotetext{
${ }^{4}$ https://elbe.hki-jena.de/fungifun/
} 
(per $1 \mathrm{~L}: 3 \mathrm{~g}$ beef extract, $10 \mathrm{~g}$ soluble starch and $12 \mathrm{~g}$ agar at $\mathrm{pH}$ 7.5). In this assay, iodine forms a black/dark blue complex with starch, but does not stain sugars, resulting in a yellowish zone surrounding the colony where starches have been metabolized. $\sim 10,000$ conidia suspended in $20 \mu \mathrm{L} \mathrm{H}_{2} \mathrm{O}$ were pipetted onto the center of the starch plates. Plates were incubated at $32^{\circ} \mathrm{C}$ for $39 \mathrm{~h}$ then flooded with iodine. This experiment was performed in triplicate. Plates were imaged with the Interscience Scan 1200.

\section{Proteolytic Assay}

To examine the proteolytic activity of isolates, we performed an established zone of clearance assay (Rajamani and Hilda, 1987). Briefly, $\sim 100,000$ conidia suspended in $20 \mu \mathrm{L} \mathrm{H}_{2} \mathrm{O}$ were inoculated onto media consisting of $2.5 \mathrm{~g}$ agar, $2.5 \mathrm{~g}$ powdered skim milk and $125 \mathrm{~mL}$ distilled $\mathrm{H}_{2} \mathrm{O}$. Ten biological replicates of each strain were grown at $32^{\circ} \mathrm{C}$ for $72 \mathrm{~h}$, at which time the zone of clearance was measured at two independent locations using digital calipers. The size of the zone is positively associated with higher proteolytic activity (Rajamani and Hilda, 1987). A 2talied $t$-test was conducted to compare zone of clearance size between isolates.

\section{Growth Rate of A. oryzae 14160 and A. oryzae RIB 40}

We compared the growth rate of A. oryzae 14160 and RIB 40 on starch agar (as defined above), PDA, rice agar and soy agar. PDA (Fisher Scientific DF0013) was prepared according to manufacturer instructions. Rice agar was prepared using $75 \mathrm{~g}$ of short grain sushi rice which was powered in a dry blender, and $15 \mathrm{~g}$ agar in $1 \mathrm{~L}$ distilled $\mathrm{H}_{2} \mathrm{O}$. For the soybean agar, dried soybeans were soaked in $\mathrm{H}_{2} \mathrm{O}$ for $24 \mathrm{~h}$, then $30 \mathrm{~g}$ soybean and $15 \mathrm{~g}$ agar were pureed together in $1 \mathrm{~L}$ distilled $\mathrm{H}_{2} \mathrm{O}$. All media was sterilized and cooked via autoclaving, and $30 \mathrm{~mL}$ of media was plated into petri dishes. $20 \mu \mathrm{L}$ of 500,000 conidia/mL spore solutions were pipetted onto the center of each plate. All growth rate experiments were performed in 10 replicates. Plates were incubated at $32^{\circ} \mathrm{C}$ for $39 \mathrm{~h}$. Because colony morphology is not always uniformly circular, colony diameter was measured at two independent points for each colony using digital calipers. The average colony diameter for each plate value was used for the statistical analysis. One-way ANOVA was used to test the null hypothesis that growth rate did not differ between culture media for each isolate. $T$-tests were performed on each culture media to test the null hypothesis that $A$. oryzae 14160 and RIB 40 growth rates did not differ.

\section{RESULTS}

\section{DNA Sequencing Data}

We generate 830,485 ONT reads totaling $\sim 5$ billion bp with an average and median read length of 6,065 and 4,092 bp, respectively, and an N50 value of 10,289 bp (Supplementary Figure 1). $96.69 \%$ of ONT reads mapped to the A. oryzae 14160 assembly. For Illumina data, a total of 17,286,313 paired-end reads were retained after adapter trimming, quality trimming, and error correction.

\section{Phylogenetic Analysis}

We performed phylogenetic analysis to investigate the relationship of $A$. oryzae 14160 in relation to the eight major clades of $A$. oryzae (Watarai et al., 2019). Specifically, we identified 243,486 SNPs from publicly available Illumina whole-genome sequencing data from $91 \mathrm{~A}$. oryzae isolates and A. flavus NRRL 3357 (Gibbons et al., 2012; Nierman et al., 2015; Watarai et al., 2019) (see Methods for NCBI BioProject accession numbers). A phylogenetic tree was inferred from the alignment of SNPs with RAxML (Stamatakis, 2014) and the tree was rooted by A. flavus NRRL 3357. Our results were identical with Watarai et al. (2019) in showing that A. oryzae isolates group into eight major clades (A-H) (Figure 1). A. oryzae 14160 was nested within clade A, which contained 26 other A. oryzae isolates from Japan (Watarai et al., 2019), and A. oryzae RIB 40 was nested within clade F (Figure 1). To explore the impact of SNP marker linkage on inferred evolutionary relationship of isolates, we also conducted phylogenetic analysis using smaller subsets of SNP markers separated by a minimum physical distance of 4 and $10 \mathrm{~Kb}$. For all analyses, the clade compositions were identical (Figure 1 and Supplementary Figures 2, 3).

\section{A. oryzae 14160 Genome Assembly and Annotation}

De novo hybrid genome assembly of $A$. oryzae 14160 was performed with the MaSuRCA assembler (Zimin et al., 2013). The A. oryzae 14160 genome was assembled into 24 scaffolds with a cumulative length of $36.5 \mathrm{Mb}$, largest scaffold length of $4.15 \mathrm{Mb}$, an $\mathrm{N} 50$ of $2.21 \mathrm{Mb}$ and an N90 of $937 \mathrm{~Kb}$. Genome assessment was performed with Quast (Gurevich et al., 2013) and showed a $95 \%$ genome fraction compare to the reference A. oryzae RIB40 genome. Genome completeness was evaluated with BUSCO (Simao et al., 2015) and showed 99\% recovery of complete BUSCO genes with $(0.1 \%$ fragmented BUSCO genes and $0.9 \%$ missing BUSCO genes). Both analyses indicate that the A. oryzae 14160 genome assembly is of high quality in terms of contiguity and accuracy.

Genome prediction and annotation of A. oryzae 14160 was performed using the Funannotate pipeline which relies on Augustus for gene prediction (Stanke and Waack, 2003). Using this pipeline, we predicted 11,852 protein-coding genes in A. oryzae 14160 , which is similar to the RIB 40 genome (12,074 protein-coding genes). The gene set was assessed for completeness using BUSCO, resulting in 93\% completeness with only $5 \%$ fragmented genes and $1.7 \%$ missing genes.

\section{A. oryzae 14160 and RIB40 Chromosomal Alignment}

We used Mummer to investigate the synteny between the 24 A. oryzae 14160 scaffolds and the eight $A$. oryzae RIB 40 chromosomes. contig_8, contig_10, contig_20, contig_15, contig_16, and contig_7 mapped to chromosome 1, contig_14 and part of contig_5 mapped to chromosome 2, contig_1 and contig_13 mapped to chromosome 3, contig_2, contig_22, contig_23, and contig_24 mapped to chromosome 4, contig_12, contig_11, contig_19, and contig_25 mapped to chromosome 


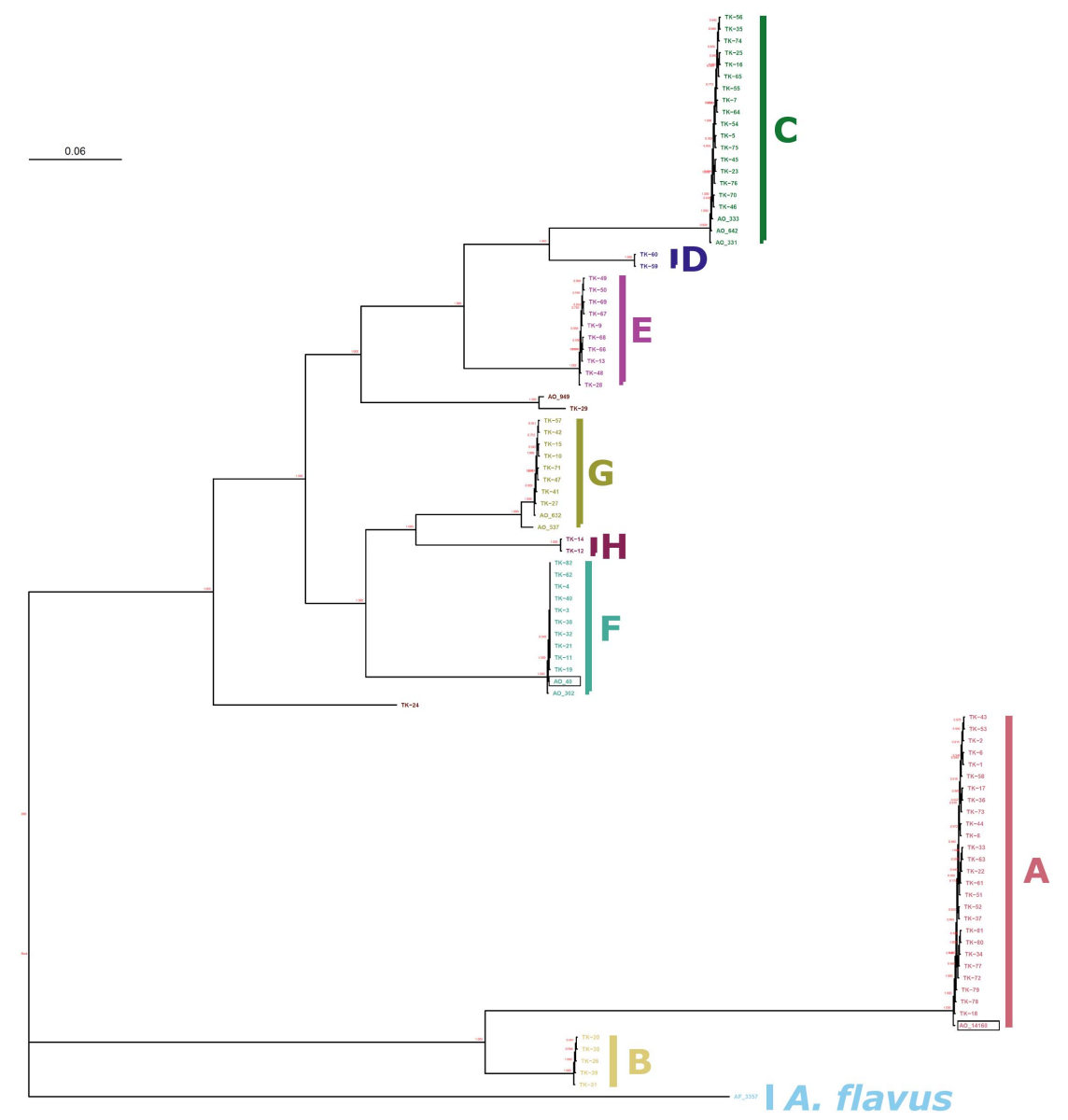

FIGURE 1 | Phylogenetic relationship of $A$. oryzae 14160 and RIB 40. Maximum-likelihood phylogeny of 91 A. oryzae isolates and $A$. flavus NRRL 3357 . Clades are labeled with respect to the nomenclature used by Watarai et al. (2019). A. oryzae 14160 and A. oryzae RIB 40 are outlined with a black back and belong to clades $F$ and $\mathrm{A}$, respectively.

5, contig_7 mapped to chromosome 6, contig_6 and contig_18 mapped to chromosome 7 , and contig_4 and contig_3 mapped to chromosome 8 (Figure 2 ). Nearly all contigs from A. oryzae 14160 mapped uniquely to their respective RIB40 chromosome with exception of contig_5 and contig_9, which aligned to multiple chromosomes (Figure 2).

\section{Alpha-Amylase Synteny}

We identified three distinct loci containing the alpha-amylase encoding gene in the A. oryzae 14160 genome (Figure 3) (alpha-amylase gene IDs = contig_14: FUN_004371, contig_5: FUN_008670, and contig_7 = FUN_010081). The alphaamylase locus on A. oryzae 14160 contig_14 displayed conserved synteny with the $A$. oryzae RIB 40 chromosome 2 alpha-amylase locus (Figure $\mathbf{3 A}$ ). In addition to the chromosome 2 locus, A. oryzae RIB 40 harbors alphaamylase loci on chromosomes 3 and 5, however, we did not observe shared synteny between these loci in $A$. oryzae 14160 (Figures 3B,C).

The $A$. oryzae 14160 genome contained alpha-amylase loci on contig_5 and contig_7, which map to A. oryzae RIB40 chromosomes 2 and 6, respectively (Figure 2). The contig_5 locus contains 9 predicted genes not present on the A. oryzae RIB 40 genome nested within two syntenic genes (FUN_008665 and FUN_008675) (Figure 3B). Importantly, this locus was assembled identically in the A. oryzae 14160 MaSuRCA and Canu assemblies, while the SPAdes assembly was also identical with the exception of an assembly break that did not include an additional copy of the genes FUN_008673 and FUN_008668 (Figure 3B). Additionally, we identified several long ONT reads $(>30 \mathrm{~Kb})$ that spanned the alpha-amylase gene as well as up-stream and/or down-stream flanking region genes. Specifically, one ONT read (ONT read ID 07fba920-71fc460e-ba46-46ebda40194a) spanned FUN_008668-FUN_008672 (contig_5:1,224,311-1,258,020) while another (ONT read ID a63546c6-6767-4fcf-9051-9393777f6572) spanned FUN_008669FUN_008673 (contig_5: 1,231,983-1,265,735). We also identified a $\sim 9 \mathrm{~Kb}$ region nearly identical to the $\mathrm{Tc} 1 /$ mariner class transposable element directly upstream of FUN_008671 (contig_5: 1,240,697-1,245,364), which has been previously observed in the some alpha-amylase loci in A. oryzae strains (Hunter et al., 2011). 


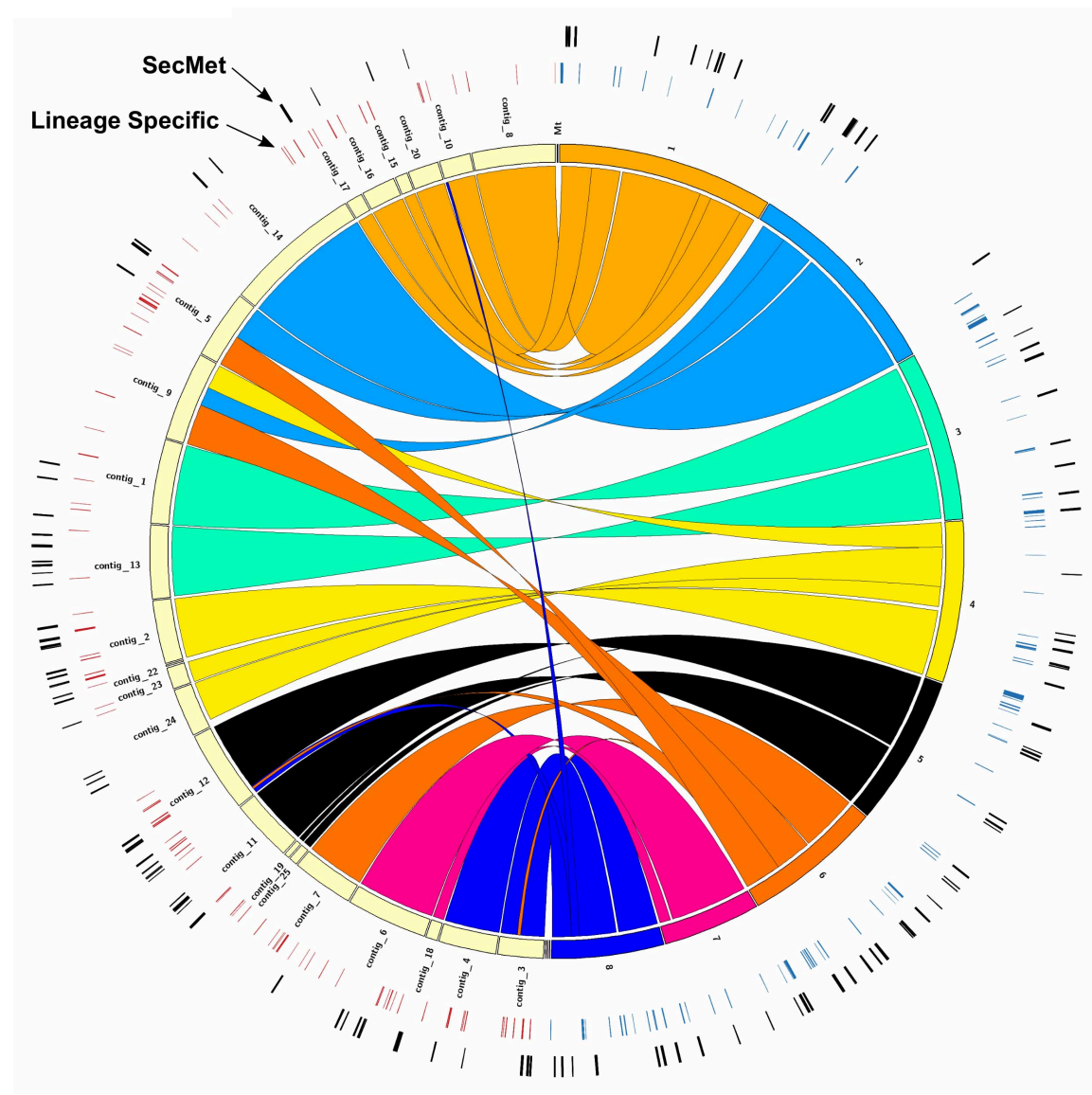

FIGURE 2 | Genome architecture between A. oryzae 14160 and RIB 40. Circos plot displaying similarity between the $A$. oryzae 14160 (left half of circle) and RIB 40 (right half of circle) genomes. The innermost ring represents $A$. oryzae 14160 scaffold ID or $A$. oryzae RIB 40 chromosome ID, and colored regions connecting the two represent regions with high sequence similarity ( $\geq 95 \%$, length $\geq 10 \mathrm{~Kb}$ ). The outer circles represent lineage specific genes and genes located within putative secondary metabolite encoding gene clusters (SecMet). The three small contigs to the left of A. oryzae RIB 40 chromosome 8 are AP007160, AP007177, and AP007156 (from left to right).

The A. oryzae 14160 alpha-amylase locus on contig_7 mapped to $A$. oryzae RIB 40 chromosome 6 , and displayed conserved synteny for the majority of the locus albeit without the alpha-amylase encoding gene (FUN_010081) and the upstream flanking gene (FUN_010082) in A. oryzae RIB 40 (Figure 3C). We identified two long ONT reads that spanned the genomic regions harboring FUN_010076-FUN_010082 and FUN_010076-FUN_010083, respectively (ONT read ID 05e9d1bc-f5c1-4a64-85a2-6d5df71f58db = contig_7: 977,243-1,011,315 and ONT read ID f5434ebf-bbda-48dd8f74-28ed565a5c6b = contig_7: 977,137-1,018,308). Again, we identified the Tc1/mariner class transposable element directly upstream of FUN_010082 (contig_7: 1,013,761-1,018,433). These results suggest convergent evolution of alpha-amylase duplication in the A. oryzae 14160 and RIB 40 genomes.

\section{Alpha-Amylase Upstream Region Conservation}

To investigate if differences in the regulatory region of the alphaamylase genes corresponded to differences in amylase activity or starch metabolism, we aligned the $1 \mathrm{~Kb}$ upstream region of the six alpha-amylase genes. We observed only two polymorphic sites (Supplementary Figure 4). Specifically, we observed a transversion from A to $\mathrm{C}$ at one position in the A. oryzae 14160 alpha-amylase gene on chromosome 6 (contig_7). In another position, we observed a transversion from $\mathrm{T}$ to $\mathrm{A}$ in the A. oryzae RIB 40 chromosome 2 copy. These results suggest that it is unlikely that differences in the regulatory regions of the alphaamylase genes contribute to differences in amylase activity or starch metabolism.

\section{Single Nucleotide Polymorphism Analysis}

We used freebayes and vcftools to identify high quality SNPs in A. oryzae 14160 relative to the A. oryzae RIB 40 reference genome. We identified 130,311 SNPs in A. oryzae $14160(\sim 1$ SNPs per $290 \mathrm{bp})$ and used SnpEff to annotate and predict the putative impact of these SNPs (Cingolani et al., 2012). $\sim 35 \%$ of SNPs were located within the coding region of genes. Of this subset, $55.64 \%$ were silent variants, 
A

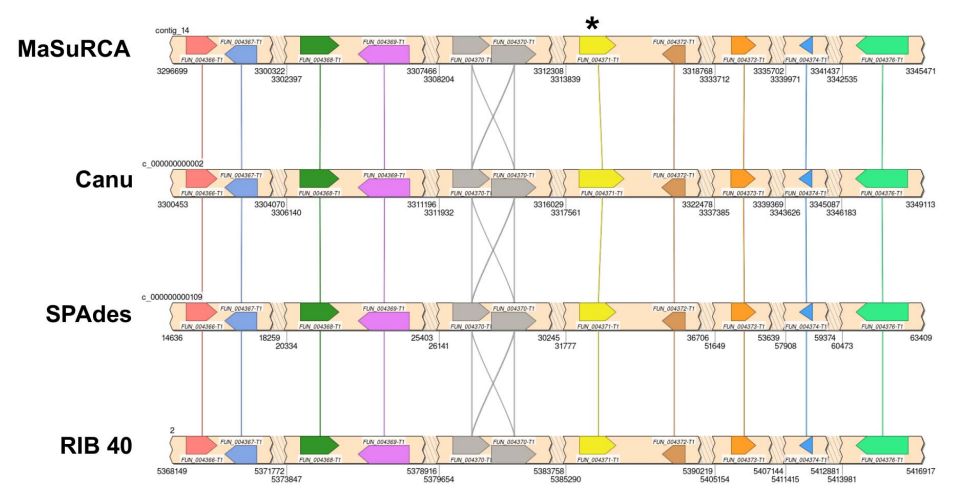

B

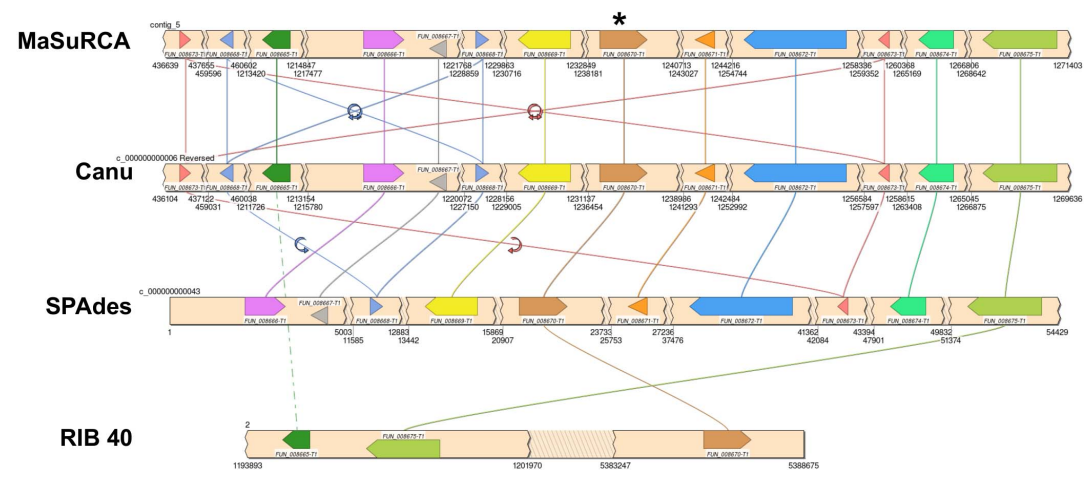

C

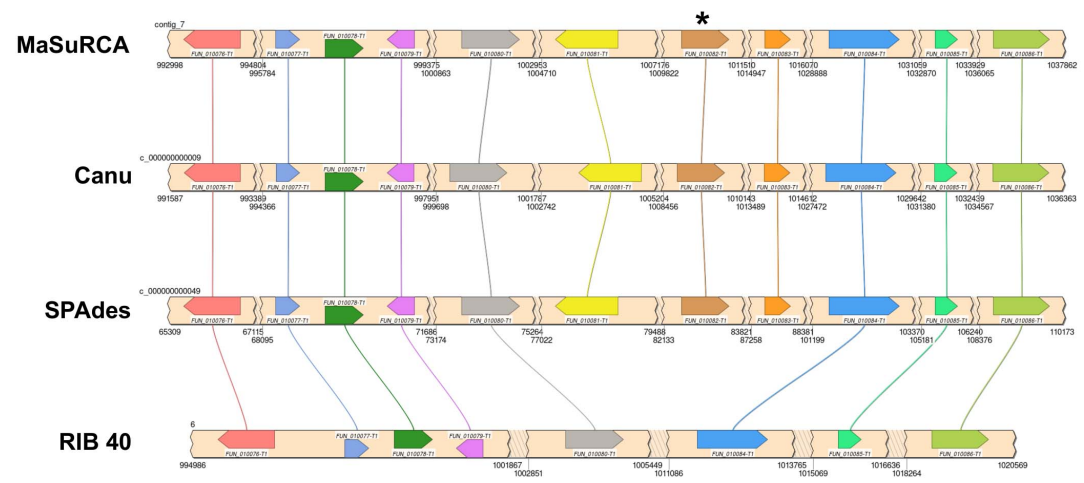

FIGURE 3 | Independent evolution of alpha-amylase duplication in A. oryzae 14160. Synteny analysis of the conserved A. oryzae 14160 alpha-amylase locus on chromosome 2 (A), the non-syntenic alpha-amylase locus on chromosome 2 (B), and the non-syntenic alpha-amylase locus on chromosome 6 (C) from three independent genome assemblies of $A$. oryzae 14160 (MaSuRCA, Canu, and SPAdes) and the reference RIB 40 genome. For each locus, gene direction is indicated by pointed ends, jagged edges indicate a genomic region without protein-coding genes that is skipped for figure clarity. Gene names are with respect to the primary A. oryzae 14160 annotation [generated from the MaSuRCA assembly (Zimin et al., 2013) and annotated with the Funannotate pipeline]. Scaffold ID or chromosome number and coordinates are labeled. "*” represents the alpha-amylase encoding genes. Lines connecting genes indicated conservation, and flip arrows represent a change in gene direction (B). Locus schematics were generated with SimpleSynteny (Veltri et al., 2016).

$43.78 \%$ were missense variants, and $0.58 \%$ were nonsense variants. We quantified the missense variant rate in each gene which ranged from 0 (no missense variants) to 0.039 ( mean $=0.0014$, median $=0.00047)$. We considered the upper $0.05 \%$ of per-gene missense variant rates as significant $(\geq 0.0152)$, which included 60 genes (Supplementary Figure 5). This subset of genes showed no significant enrichment for GO terms. A variety of PFAM protein domains were identified in this subset of genes including transporter, protein kinase, glycosyl hydrolase, endonuclease, transposase, and transcription factor domains (Supplementary Table 1). Additionally, five genes with elevated missense variant rates were part of secondary metabolite encoding gene clusters (AO090026000589, AO090102000459, AO090103000221, 

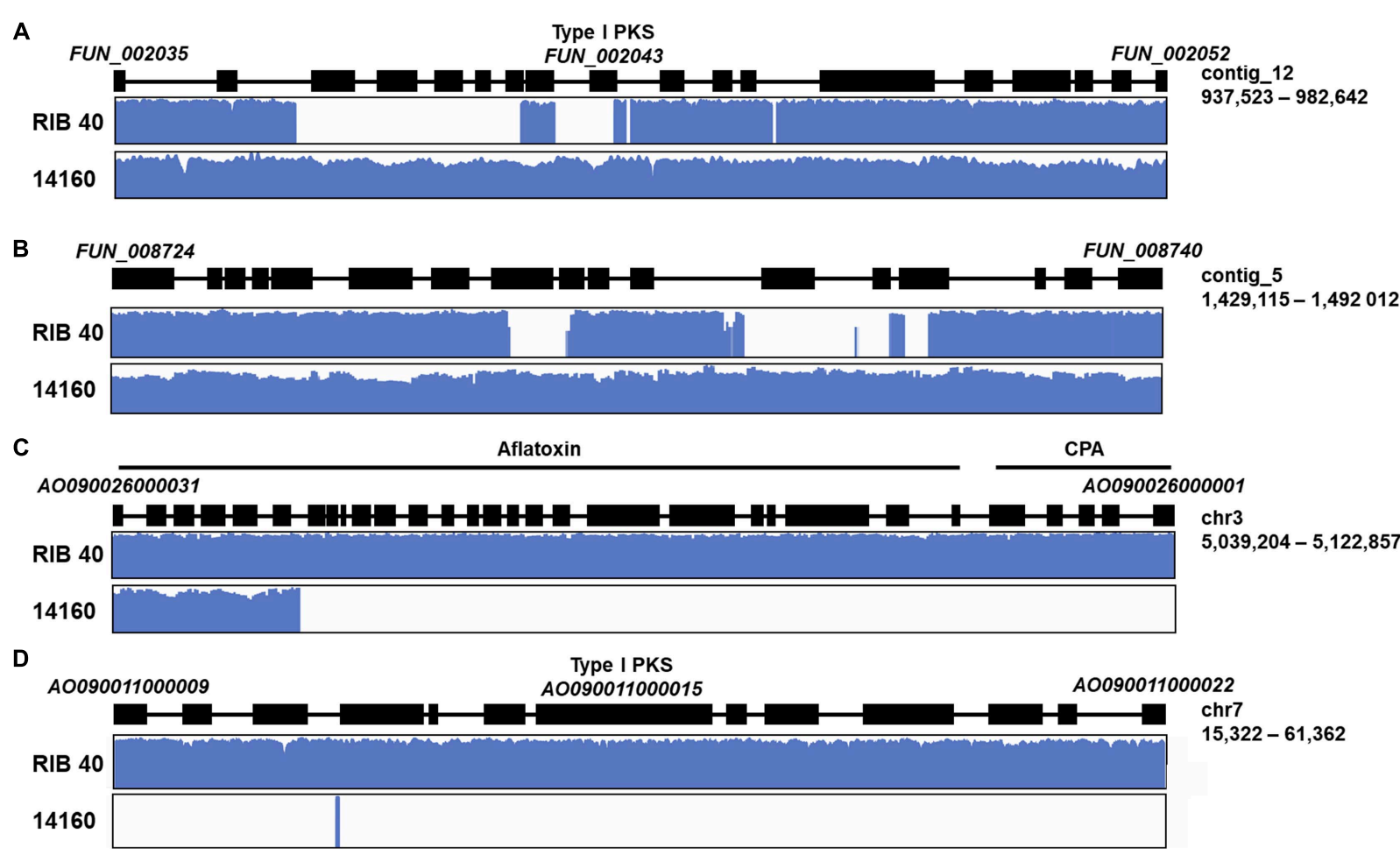

FIGURE 4 | Examples of lineage specific genes in secondary metabolite encoding gene clusters. Lineage specific genes with functional involvement in secondary metabolite production, regulation, and transport in A. oryzae 14160 (A,B), and RIB 40 (C,D). For each example, a schematic of the secondary metabolite encoding gene cluster is shown where black boxes represent genes. The first and last gene, scaffold/chromosome ID and scaffold/chromosome coordinates are labeled for each example. Graphs below the gene cluster schematic display the log10 read depth across the gene cluster for each isolate.

and A0090701000565), although we did not observe an overrepresentation of genes in secondary metabolite encoding gene clusters compared to the background (Fisher's Exact Test, $p=0.60)$. Two of the genes with elevated missense variant rates neighbored one another (AO090003001358 and AO090003001359) and both genes encode proteins with predicted glycosyl hydrolases family 18 PFAM domains. Interestingly, the set of genes with elevated missense variant rates had significantly shorter coding sequences compared with the background genes $\left(\right.$ mean $_{\text {elevatedmissensevariantrategenes }}=785 \mathrm{bp}$, mean $_{\text {backgroundgenes }}=1,352 \mathrm{bp}$; Wilcoxon Signed-Rank Test, $p=1.1 \mathrm{e}-9)$.

Additionally, we examined gene enrichment analysis for the 480 genes that had one or more predicted $H I G H$ impact mutations, as defined by SnpEff (i.e., loss of stop codon, gain of stop codon, loss of start codon, splice donor variant, and splice acceptor variant) (Supplementary Table 2). We identified 10 biological process GO terms that were enriched in the genes containing HIGH impact mutations [GO:1900557 (emericellamide biosynthetic process), $p=0.0003$; GO:0050763 (depsipeptide biosynthetic process), $p=0.0006$; GO:1900555 (emericellamide metabolic process), $p=0.0006$; GO:0032774 (RNA biosynthetic process), $p=0.0013$; GO:1901336 (lactone biosynthetic process), $p=0.0013$; GO:1900560 (austinol biosynthetic process), $p=0.0028$; GO:1900558 (austinol metabolic process), $p=0.0028$; GO:1900561 (dehydroaustinol metabolic process), $p=0.0036$; GO:1900563 (dehydroaustinol biosynthetic process), $p=0.0036$; GO:0008610 (lipid biosynthetic process), $p=0.0062$ ]. In support of the functional enrichment results, we identified $H I G H$ impact mutations in 9 of the 75 secondary metabolite biosynthetic "backbone" genes [i.e., polyketide synthase (PKS), non-ribosomal peptide synthetase (NRPS), polyketide synthase/non-ribosomal peptide synthetase hybrid (PKS-NRPS), dimethylallyl tryptophan synthase (DMATS), and diterpene synthase (DTS)]. Of these genes, eight contained nonsense variants (AO090009000052, A0090009000131, A0090010000404, AO090011000328, A0090038000098, A0090038000543, AO090103000224, and $A 0090103000355)$ and one gene contained a nonstop variant (AO090001000009). AO090009000131 and AO090010000404 contained two nonsense variants, and AO090011000328 contained a nonsense variant and a splice acceptor variant. Gene length (combined exon length) was not significantly different between genes with $H I G H$ impact mutations and genes lacking $H I G H$ impact mutations ( mean $_{\text {highimpactvariantgenes }}=1,418 \mathrm{bp}$, mean $_{\text {backgroundgenes }}=1,346$ bp; Wilcoxon Signed-Rank Test, $p=0.46)$.

\section{Lineage Specific Genes}

We used control-FREEC to predict gene deletions each gene in the A. oryzae RIB 40 and A. oryzae 14160 reference genomes. Genes that were absent (copy number $=0$ ) in the mapped genome 


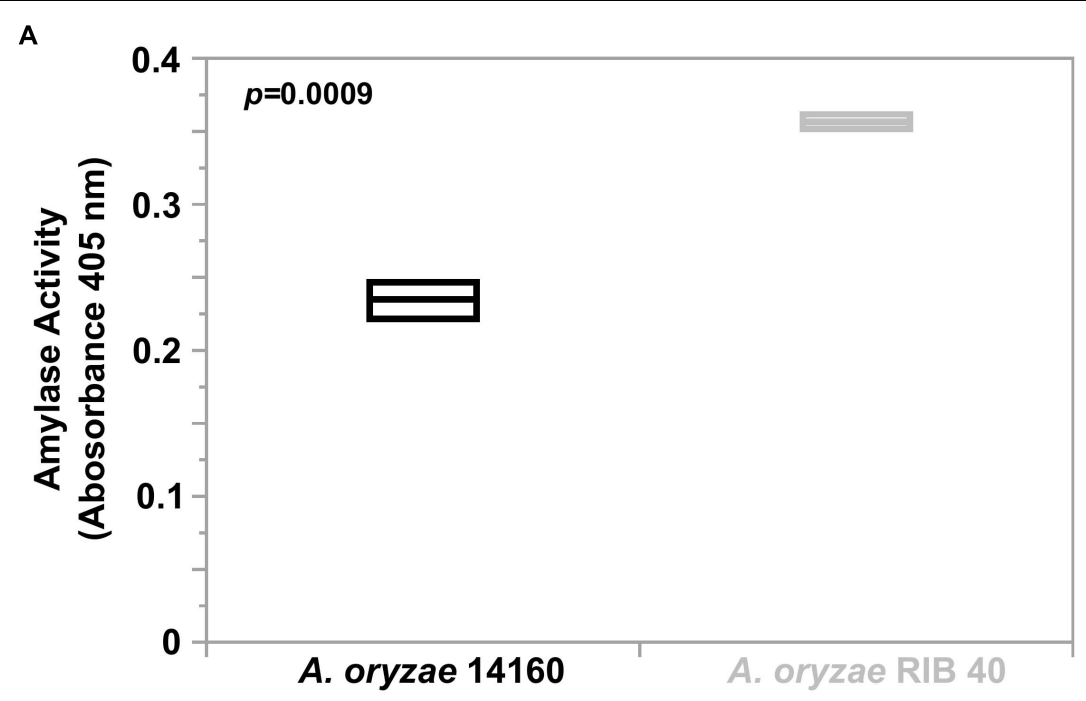

B
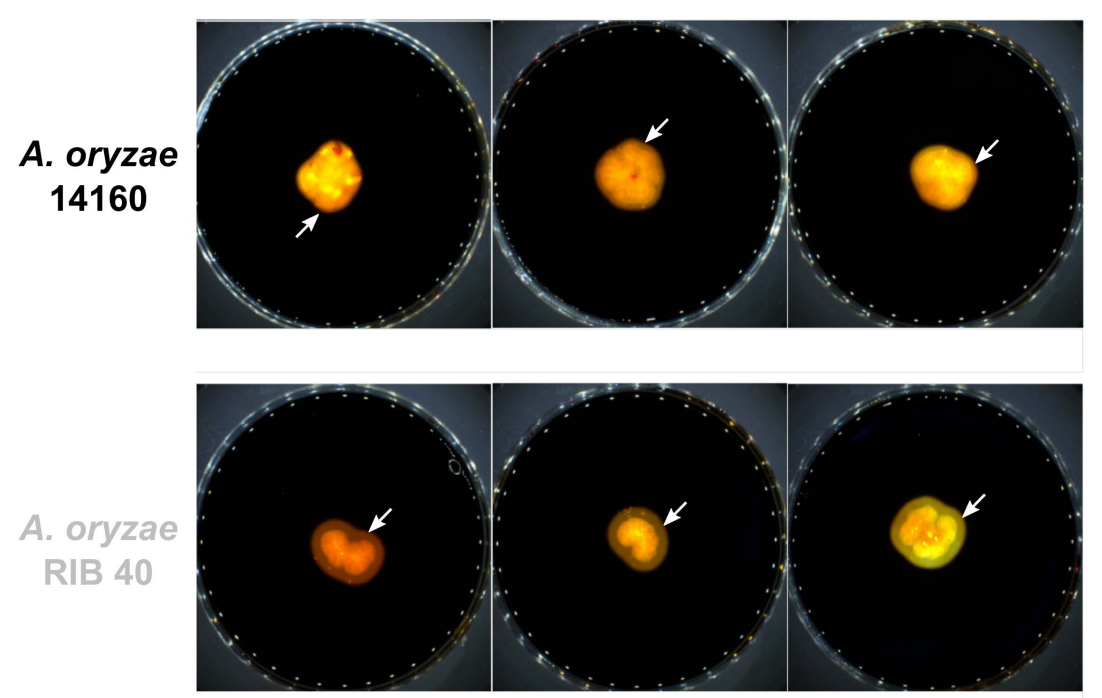

FIGURE 5 | Alpha-amylase activity in A. oryzae 14160 and RIB 40. (A) Quantitative analysis of amylase activity during solid state rice fermentation. Amylase activity was measured in triplicate using the Megazyme alpha-amylase Assay Kit. Black and Gray box plots and text represent the A. oryzae 14160 and RIB 40 isolates, respectively. (B) Qualitative iodine based amylase activity assay. Isolates were grown on starch agar and media was stained with iodine. Yellow zones surrounding the fungal colonies (white arrows) indicate amylase activity.

were considered lineage specific genes in the reference genome. Using this approach, we identified 447 and 251 genes in the A. oryzae RIB 40 and A. oryzae 14160 genomes, respectively (Figure 2 and Supplementary Tables 3, 4). Lineage specific genes were often found in clusters of neighboring genes, likely because of deletion, duplication or insertion events spanning multiple genes. For instance, $A$. oryzae RIB 40 lineage specific genes were found in 87 loci, with only 18 loci containing one gene (average $=5.1$, median $=3, \max =34$ ). The largest cluster of lineage specific genes in A. oryzae RIB 40 contained 34 genes and overlapped the aflatoxin and cyclopiazonic acid encoding gene clusters (Figure 4C). A. oryzae 14160 lineage specific genes were found in 101 loci, with 41 loci containing one gene (average $=2.5$, median $=2, \max =14$ ). The largest lineage specific gene cluster in $A$. oryzae 14160 contained 14 genes (FUN_008412-FUN_008425).

A. oryzae RIB 40 lineage specific genes were functionally enriched for the biological process GO terms "secondary metabolite biosynthetic process" $(p=7.87 \mathrm{e}-10)$, and "sterigmatocystin biosynthetic process" ( $p=1.35 \mathrm{e}-5)$, and the molecular function GO terms "oxidoreductase activity, acting on paired donors, with incorporation or reduction of molecular oxygen" ( $p=1 \mathrm{e}-6)$, "heme binding" ( $p=1.24 \mathrm{e}-5)$, "iron ion binding" ( $p=1.91 \mathrm{e}-5)$, and "electron carrier activity" $(p=2.21 \mathrm{e}-5)$. Because we observed an overrepresentation of lineage specific genes involved in secondary metabolism in A. oryzae RIB 40, we also tested whether this trend was present in A. oryzae 14160 lineage specific genes. For this analysis, we 
tested whether genes annotated within secondary metabolite encoding gene clusters (as annotated by antiSMASH; Medema et al., 2011) were overrepresented in the lineage specific genes vs. the non-lineage specific genes. Indeed, we observed an overrepresentation of genes involved in secondary metabolism in the $A$. oryzae 14160 lineage specific genes (Fisher's exact test, $p=0.0013)$. Specifically, $11.5 \%$ of $A$. oryzae 14160 lineage specific genes were annotated within secondary metabolite encoding gene clusters compared to $5.6 \%$ in the background, and these genes fell within 11 independent secondary metabolite encoding gene clusters. Using the same approach, we again identified an enrichment of genes in secondary metabolite encoding gene clusters in $A$. oryzae RIB 40 (Fisher's exact test, $p=2 \mathrm{e}-11$ ).

\section{A. oryzae 14160 and A. oryzae RIB 40 Amylase Activity, Proteolytic Activity, and Growth Rate}

Because we observed widespread genomic variation between A. oryzae 14160 and RIB 40, we were interested in how this variation may affect phenotype. Thus, we measured and compared amylase activity and growth rate of both strains. We hypothesized that alpha-amylase activity would be similar between $A$. oryzae 14160 and RIB 40 because both strains possess three copies of the alpha-amylase encoding gene (Figure 3 ). Interestingly, quantitative analysis of amylase activity during solid-state rice fermentation, and qualitative amylase activity on starch agar showed that $A$. oryzae RIB 40 produces higher levels of amylase (Figure 5). However, A. oryzae 14160 and RIB 40 did not significantly differ in their proteolytic activity (Supplementary Figure 6).

Both strains showed significantly different growth rates between media types (A. oryzae 14160: Oneway Anova, d.f. $=3$, F-ratio $=31.4, p=1.73 \mathrm{e}-10$ and $A$. oryzae RIB 40: Oneway Anova, d.f. $=3$, F-ratio $=22.8, p=1.07 \mathrm{e}-8)$ (Supplementary Figure 7). A. oryzae 14160 grew fastest on PDA and starch, while growing significantly slower on soy, followed by rice (Supplementary Figure 7A). A. oryzae RIB 40 grew fastest on PDA, while growth on starch, rice and soy were not significantly different from one another (Supplementary Figure 7B). We also compared the growth rate of $A$. oryzae 14160 vs. RIB 40 for each media type. A. oryzae 14160 grew significantly faster on soy ( $t$-test, t-ratio $=-2.3, p=0.03)$ and starch ( $t$-test, t-ratio $=-5.1$, $p=0.00003$ ) while A. oryzae RIB 40 grew significantly faster on PDA ( $t$-test, t-ratio $=2.3, p=0.017)$ and rice $(t$-test, $t$-ratio $=2.8$, $p=0.006)$ (Figure 6).

\section{DISCUSSION}

Here, we used long-read ONT and short-read Illumina sequencing data to assemble an accurate and highly contiguous genome of $A$. oryzae 14160 . To date, only four $A$. oryzae isolates have genome assemblies comprised of fewer than 30 scaffolds (RIB40, BP2-1, BCC7051, and TK-29) (Thammarongtham et al., 2018; Watarai et al., 2019; Jeon et al., 2020). These isolates belong to clade F (RIB 40), clade E (BCC7051), clade BP2-1 (BP2-1), and a smaller clade closely related the BP2-1 clade (TK-29) (Watarai

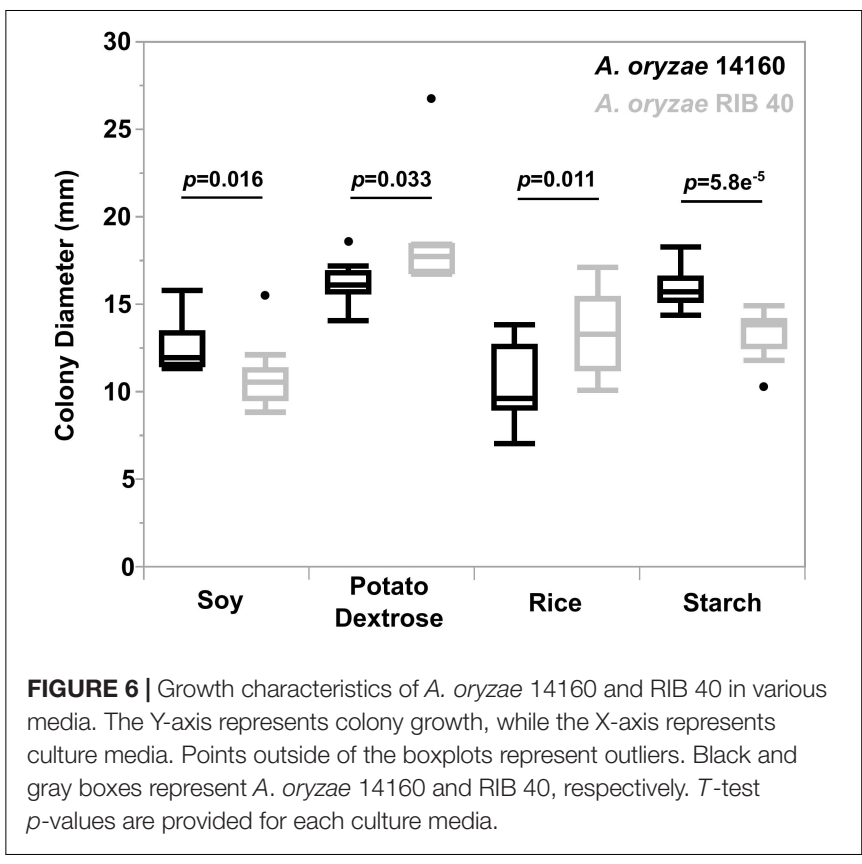

et al., 2019). Our phylogenetic analysis revealed that A. oryzae 14160 is part of clade A, and thus represents the first highly contiguous genome assembly from this group. Importantly, high quality genome assemblies from representative isolates across clades will enable comparative genomic analysis of structural variants, as we have demonstrated here with our synteny analysis of the alpha-amylase loci (Figure 3).

The assembly and annotation of the A. oryzae 14160 genome enabled in depth comparative genomic analysis with the complete chromosome assembly of the A. oryzae RIB 40 reference genome. We conducted several analyses to identify genes with divergent patterns (i.e., relative abundance of missense variants, putative impact of variants, and gene presence/absence) in the A. oryzae 14160 and RIB 40 genomes. Collectively, these analyses revealed that genes with involvement in secondary metabolism were highly variable (Figures 2, 4). For instance, we observed a largescale deletion event of the aflatoxin biosynthetic gene cluster that includes more than half of the cluster and the neighboring cyclopiazonic acid encoding gene cluster (Figure 4C). Large-scale chromosomal deletions of the aflatoxin encoding gene cluster have been previously characterized in A. oryzae isolates (Lee et al., 2006; Tominaga et al., 2006; Chang and Ehrlich, 2010; Alshannaq et al., 2018). Interestingly, a number of independent loss of function variants have also been observed in A. oryzae strains resulting in their inability to produce aflatoxin. This observation indicates the loss of aflatoxin has independently evolved in different $A$. oryzae clades, perhaps to reallocate the high energy demands required to produce this secondary metabolite into primary metabolism (Gibbons, 2019).

In addition we observed a number of high impact variants and gene presence polymorphisms in several putative secondary metabolite backbone encoding genes whose products are not as well-characterized as aflatoxin and cyclopiazonic acid (Figure 4). For example, FUN_002043 encodes a type I iterative polyketide 
synthetase and is absent in the A. oryzae RIB 40 genome, and a large secondary metabolite encoding gene cluster on A. oryzae chromosome 7 is entirely absent from the A. oryzae 14160 genome (Figure 4A). These results are consistent with observations in Aspergillus species that show secondary metabolite encoding gene clusters are highly variable both between and within species (Gibbons et al., 2012; Ehrlich and Mack, 2014; Lind et al., 2017; Alshannaq et al., 2018; Zhao and Gibbons, 2018; Drott et al., 2020; Kjaerbolling et al., 2020; Steenwyk et al., 2020). For example, genomic analysis of three Aspergillus nidulans genomes revealed more than 70 secondary metabolite encoding gene clusters in each genome while nine of these clusters displayed presence/absence polymorphisms (Drott et al., 2020). Similarly, we previously observed a polymorphic locus with two distinct secondary metabolite gene clusters in A. oryzae and A. flavus (Gibbons et al., 2012).

Alpha-amylase is an enzyme that hydrolyzes the alpha-Dglyosidic bond in starch to produce dextrin, and the high production of this carbohydrate metabolizing enzyme is, perhaps, A. oryzae's most important industrial characteristic. Alphaamylase copy number varies from one to four in $A$. oryzae isolates and these gene duplication events likely derive from the Tc1/mariner like transposable element that flanks this locus (Hunter et al., 2011; Watarai et al., 2019). Hunter et al. (2011) provided evidence for at least three independent duplication events of the alpha-amylase locus from the ancestral chromosome 2 copy. Interestingly, we also observed conservation of the alphaamylase locus on chromosome 2 in A. oryzae 14160 (Figure 3), which is also conserved in the A. flavus NRRL 3357 genome (Nierman et al., 2015). However, we did not observe alphaamylase copies on chromosomes 3 and 5 as in the A. oryzae RIB 40 genome. Instead, we identified an additional copy of the alpha-amylase locus on chromosome 2 and chromosome 6, providing further evidence for convergent evolution of alphaamylase duplication in A. oryzae. The independent duplication of alpha-amylase indicates that artificial selection for increased amylase production was very strong during the domestication of A. oryzae.

Because we observed extensive genome variation between A. oryzae 14160 and RIB 40 we investigated how these strains differed phenotypically. First, we measure amylase activity using two independent assays. Both assays showed that $A$. oryzae RIB 40 produces greater levels of alpha-amylase (Figure 5). This observation was somewhat surprising considering the genomes of both strains contain three copies of the alphaamylase encoding gene, and the upstream regions of these genes are nearly identical (Supplementary Figure 4). However, a study that generated single, double, and triple disruptant mutants of the three alpha-amylase encoding genes in RIB 40 revealed that the contribution of amylase gene and protein expression was not equal between the three copies (Nemoto et al., 2012). More specifically, amyA (the conserved alphaamylase copy on chromosome 2) contributed least to amylase production. Consequently, the newly duplicated copies of alphaamylase may also dominate amylase expression in other A. oryzae isolates, and chromosomal location of alpha-amylase paralogs may influence their gene expression. For instance, position effect variegation was observed in A. nidulans where a translocation of the developmental regulator $\operatorname{brl} A$ resulted a conidiophore that remained as a stiff hyphae and did not develop a vesicle, sterigmata, and conidia (Clutterbuck, 1970). Similarly, the chromosomal position of the alpha-amylase loci in A. oryzae could potentially influence expression.

Finally, we observed differential growth preferences between A. oryzae 14160 and RIB 40. Interestingly, A. oryzae 14160 grew significantly faster on soy and starch agar, while RIB 40 grew significantly faster on PDA and rice agar. Though A. oryzae RIB 40 did not grow faster on starch agar, amylase activity was visibly greater, suggesting that growth rate may have increased during a longer incubation period. Additionally, the starch agar contained beef extract which provides a source of proteins and peptides. Thus, A. oryzae 14160 grew faster where protein content was higher (soy and starch), and RIB 40 grew faster when carbohydrates were the major available energy source (potato dextrose and rice). This observation suggests that A. oryzae 14160 is better suited for soy fermentation, while RIB 40 is better suited for rice fermentation.

\section{DATA AVAILABILITY STATEMENT}

The datasets presented in this study can be found in online repositories. The names of the repository/repositories and accession number(s) can be found below: https://www.ncbi.nlm. nih.gov/, PRJNA717291.

\section{AUTHOR CONTRIBUTIONS}

$\mathrm{KC}-\mathrm{V}$ and JG conducted genomic and statistical analysis. $\mathrm{CM}$ performed growth rate and amylase experiments. J-HY and DC maintained the cultures and extracted DNA for Illumina sequencing. LW provided the A. oryzae 14160. All authors contributed to manuscript revision and approved the submitted version.

\section{FUNDING}

This work was funded through NSF Grant 1942681 to JG which also supports $\mathrm{KC}-\mathrm{V}$.

\section{ACKNOWLEDGMENTS}

Computational analysis was conducted on the Massachusetts Green High Performance Computing Center (MGHPCC).

\section{SUPPLEMENTARY MATERIAL}

The Supplementary Material for this article can be found online at: https://www.frontiersin.org/articles/10.3389/fmicb.2021. 691296/full\#supplementary-material 


\section{REFERENCES}

Abbo, S., Pinhasi van-Oss, R., Gopher, A., Saranga, Y., Ofner, I., and Peleg, Z. (2014). Plant domestication versus crop evolution: a conceptual framework for cereals and grain legumes. Trends Plant Sci. 19, 351-360. doi: 10.1016/j.tplants. 2013.12.002

Alshannaq, A. F., Gibbons, J. G., Lee, M. K., Han, K. H., Hong, S. B., and Yu, J. H. (2018). Controlling aflatoxin contamination and propagation of Aspergillus flavus by a soy-fermenting Aspergillus oryzae strain. Sci. Rep. 8:16871. doi: 10.1038/s41598-018-35246-1

Altschul, S. F., Madden, T. L., Schaffer, A. A., Zhang, J., Zhang, Z., Miller, W., et al. (1997). Gapped BLAST and PSI-BLAST: a new generation of protein database search programs. Nucleic Acids Res. 25, 3389-3402. doi: 10.1093/nar/25.17.3389

Aouizerat, T., Gutman, I., Paz, Y., Maeir, A. M., Gadot, Y., Gelman, D., et al. (2019). Isolation and characterization of live yeast cells from ancient vessels as a tool in bio-archaeology. Mbio 10:e00388-19. doi: 10.1128/mBio.00388-19

Aouizerat, T., Maeir, A. M., Paz, Y., Gadot, Y., Szitenberg, A., Alkalay-Oren, S., et al. (2020). Isolation and characterization of live yeast cells from ancient clay vessels. Bio Protoc. 10:e3473. doi: 10.21769/BioProtoc.3473

Bankevich, A., Nurk, S., Antipov, D., Gurevich, A. A., Dvorkin, M., Kulikov, A. S., et al. (2012). SPAdes: a new genome assembly algorithm and its applications to single-cell sequencing. J. Comput. Biol. 19, 455-477. doi: 10.1089/cmb.2012. 0021

Boeva, V., Popova, T., Bleakley, K., Chiche, P., Cappo, J., Schleiermacher, G., et al. (2012). Control-FREEC: a tool for assessing copy number and allelic content using next-generation sequencing data. Bioinformatics 28, 423-425. doi: 10.1093/bioinformatics/btr670

Cavalieri, D., McGovern, P. E., Hartl, D. L., Mortimer, R., and Polsinelli, M. (2003). Evidence for S. cerevisiae fermentation in ancient wine. J. Mol. Evol. 57 Suppl 1, S226-S232. doi: 10.1007/s00239-003-0031-2

Chang, P. K., and Ehrlich, K. C. (2010). What does genetic diversity of Aspergillus flavus tell us about Aspergillus oryzae? Int. J. Food Microbiol. 138, 189-199. doi: 10.1016/j.ijfoodmicro.2010.01.033

Cingolani, P., Platts, A., Wang, L. L., Coon, M., Nguyen, T., Wang, L., et al. (2012). A program for annotating and predicting the effects of single nucleotide polymorphisms, SnpEff: SNPs in the genome of Drosophila melanogaster strain w(1118); iso-2; iso-3. Fly 6, 80-92. doi: 10.4161/fly.19695

Clutterbuck, A. J. (1970). A variegated position effect in Aspergillus nidulans. Genet. Res. 16, 303-316. doi: 10.1017/s0016672300002561

Danecek, P., Auton, A., Abecasis, G., Albers, C. A., Banks, E., DePristo, M. A., et al. (2011). Genomes project analysis, the variant call format and VCFtools. Bioinformatics 27, 2156-2158. doi: 10.1093/bioinformatics/btr330

Davis, M. P., van Dongen, S., Abreu-Goodger, C., Bartonicek, N., and Enright, A. J. (2013). Kraken: a set of tools for quality control and analysis of high-throughput sequence data. Methods 63, 41-49. doi: 10.1016/j.ymeth.2013.06.027

Delcher, A. L., Kasif, S., Fleischmann, R. D., Peterson, J., White, O., and Salzberg, S. L. (1999). Alignment of whole genomes. Nucleic Acids Res. 27, 2369-2376. doi: 10.1093/nar/27.11.2369

Drott, M. T., Bastos, R. W., Rokas, A., Ries, L. N. A., Gabaldon, T., Goldman, G. H., et al. (2020). Diversity of secondary metabolism in aspergillus nidulans clinical isolates. $m$ Sphere 5:e0156-20. doi: 10.1128/mSphere.00156-20

Dumas, E., Feurtey, A., Rodriguez de la Vega, R. C., Prieur, S. Le, Snirc, A., Coton, M., et al. (2020). Independent domestication events in the blue-cheese fungus Penicillium roqueforti. Mol. Ecol. 29, 2639-2660. doi: 10.1111/mec.15359

Ehrlich, K. C., and Mack, B. M. (2014). Comparison of expression of secondary metabolite biosynthesis cluster genes in Aspergillus flavus, A. parasiticus, and A. oryzae. Toxins (Basel) 6, 1916-1928. doi: 10.3390/toxins6061916

Fuwa, H. (1954). A new method for microdetermination CF amylase activity by the use of amylose as the substrate. J. Biochem. 41, 583-603. doi: 10.1093/ oxfordjournals.jbchem.a126476

Gallone, B., Steensels, J., Prahl, T., Soriaga, L., Saels, V., Herrera-Malaver, B., et al. (2016). Domestication and divergence of Saccharomyces cerevisiae beer yeasts. Cell 166, 1397-1410.e16.

Garrison, E., and Marth, G. (2012). Haplotype-based variant detection from short-read sequencing. arXiv [Preprint] arXiv: 1207.3907v2,

Geiser, D. M., Pitt, J. I., and Taylor, J. W. (1998). Cryptic speciation and recombination in the aflatoxin-producing fungus Aspergillus flavus. Proc. Natl. Acad. Sci. U.S.A. 95, 388-393. doi: 10.1073/pnas.95.1.388
Gibbons, J. G. (2019). How to train your fungus. Mbio 10:e003031-19. doi: 10 . 1128/mBio.03031-19

Gibbons, J. G., and Rinker, D. C. (2015). The genomics of microbial domestication in the fermented food environment. Curr. Opin. Genet. Dev. 35, 1-8. doi: 10.1016/j.gde.2015.07.003

Gibbons, J. G., Salichos, L., Slot, J. C., Rinker, D. C., McGary, K. L., King, J. G., et al. (2012). The evolutionary imprint of domestication on genome variation and function of the filamentous fungus Aspergillus oryzae. Curr. Biol. 22, 1403-1409. doi: 10.1016/j.cub.2012.05.033

Gurevich, A., Saveliev, V., Vyahhi, N., and Tesler, G. (2013). QUAST: quality assessment tool for genome assemblies. Bioinformatics 29, 1072-1075. doi: 10.1093/bioinformatics/btt086

Hunter, A. J., Jin, B., and Kelly, J. M. (2011). Independent duplications of alphaamylase in different strains of Aspergillus oryzae. Fungal Genet. Biol. 48, 438-444. doi: 10.1016/j.fgb.2011.01.006

Jeon, J., Kim, J. A., Park, S. Y., Kim, G. W., Park, C. S., Kim, C., et al. (2020) Draft genome sequence of Aspergillus oryzae BP2-1, isolated from traditional malted rice in South Korea. Microbiol. Resour. Announc. 9, e1405-e1419. doi: 10.1128/MRA.01405-19

Jones, P., Binns, D., Chang, H. Y., Fraser, M., Li, W., McAnulla, C., et al. (2014). InterProScan 5: genome-scale protein function classification. Bioinformatics 30, 1236-1240. doi: 10.1093/bioinformatics/btu031

Kall, L., Krogh, A., and Sonnhammer, E. L. (2004). A combined transmembrane topology and signal peptide prediction method. J. Mol. Biol. 338, 1027-1036. doi: 10.1016/j.jmb.2004.03.016

Kall, L., Krogh, A., and Sonnhammer, E. L. (2007). Advantages of combined transmembrane topology and signal peptide prediction-the Phobius web server. Nucleic Acids Res. 35, W429-W432.

Katoh, K., and Standley, D. M. (2013). MAFFT multiple sequence alignment software version 7: improvements in performance and usability. Mol. Biol. Evol. 30, 772-780. doi: 10.1093/molbev/mst010

Kjaerbolling, I., Vesth, T., Frisvad, J. C., Nybo, J. L., Theobald, S., Kildgaard, S., et al. (2020). A comparative genomics study of 23 Aspergillus species from section Flavi. Nat. Commun. 11:1106. doi: omparativegenomicsstud

Koren, S., Walenz, B. P., Berlin, K., Miller, J. R., Bergman, N. H., and Phillippy, A. M. (2017). Canu: scalable and accurate long-read assembly via adaptive k-mer weighting and repeat separation. Genome Res. 27, 722-736. doi: 10.1101/ gr.215087.116

Krzywinski, M., Schein, J., Birol, I., Connors, J., Gascoyne, R., Horsman, D., et al. (2009). Circos: an information aesthetic for comparative genomics. Genome Res. 19, 1639-1645. doi: 10.1101/gr.092759.109

Larson, G., and Fuller, D. Q. (2014). The evolution of animal domestication. Annu. Rev. Ecol. Evol Syst. 45, 115-136.

Larson, G., Piperno, D. R., Allaby, R. G., Purugganan, M. D., Andersson, L., Arroyo-Kalin, M., et al. (2014). Current perspectives and the future of domestication studies. Proc. Natl. Acad. Sci. U.S.A. 111, 6139-6146. doi: 10. 1073/pnas.1323964111

Lee, M. K., Park, H. S., Han, K. H., Hong, S. B., and Yu, J. H. (2017). High molecular weight genomic DNA mini-prep for filamentous fungi. Fungal Genet. Biol. 104, 1-5. doi: 10.1016/j.fgb.2017.04.003

Lee, Y. H., Tominaga, M., Hayashi, R., Sakamoto, K., Yamada, O., and Akita, O. (2006). Aspergillus oryzae strains with a large deletion of the aflatoxin biosynthetic homologous gene cluster differentiated by chromosomal breakage. Appl. Microbiol. Biotechnol. 72, 339-345. doi: 10.1007/s00253-0050282-5

Li, H. (2018). Minimap2: pairwise alignment for nucleotide sequences. Bioinformatics 34, 3094-3100. doi: 10.1093/bioinformatics/bty191

Li, H., and Durbin, R. (2009). Fast and accurate short read alignment with BurrowsWheeler transform. Bioinformatics 25, 1754-1760. doi: 10.1093/bioinformatics/ btp324

Li, H., and Durbin, R. (2010). Fast and accurate long-read alignment with BurrowsWheeler transform. Bioinformatics 26, 589-595. doi: 10.1093/bioinformatics/ btp698

Li, H., Handsaker, B., Wysoker, A., Fennell, T., Ruan, J., Homer, N., et al. (2009). Genome project data processing. The sequence alignment/map format and SAMtools. Bioinformatics 25, 2078-2079. doi: 10.1093/bioinformatics/btp352

Lind, A. L., Wisecaver, J. H., Lameiras, C., Wiemann, P., Palmer, J. M., Keller, N. P., et al. (2017). Drivers of genetic diversity in secondary metabolic gene 
clusters within a fungal species. PLoS Biol. 15:e2003583. doi: 10.1371/journal. pbio. 2003583

Liu, L., Wang, J., Levin, M. J., Sinnott-Armstrong, N., Zhao, H., Zhao, Y., et al. (2019). The origins of specialized pottery and diverse alcohol fermentation techniques in Early Neolithic China. Proc. Natl. Acad. Sci. U.S.A. 116, 1276712774. doi: 10.1073/pnas.1902668116

Machida, M., Asai, K., Sano, M., Tanaka, T., Kumagai, T., Terai, G., et al. (2005). Genome sequencing and analysis of Aspergillus oryzae. Nature 438, 1157-1161. doi: $10.1038 /$ nature 04300

Machida, M., Yamada, O., and Gomi, K. (2008). Genomics of Aspergillus oryzae: learning from the history of Koji mold and exploration of its future. DNA Res. 15, 173-183. doi: 10.1093/dnares/dsn020

McGovern, P. E., Zhang, J., Tang, J., Zhang, Z., Hall, G. R., Moreau, R. A., et al. (2004). Fermented beverages of pre- and proto-historic China. Proc. Natl. Acad. Sci. U.S.A 101, 17593-17598. doi: 10.1073/pnas.0407921102

McKenna, A., Hanna, M., Banks, E., Sivachenko, A., Cibulskis, K., Kernytsky, A., et al. (2010). The genome analysis toolkit: a MapReduce framework for analyzing next-generation DNA sequencing data. Genome Res. 20, 1297-1303. doi: 10.1101/gr.107524.110

Medema, M. H., Blin, K., Cimermancic, P., de Jager, V., Zakrzewski, P., Fischbach, M. A., et al. (2011). antiSMASH: rapid identification, annotation and analysis of secondary metabolite biosynthesis gene clusters in bacterial and fungal genome sequences. Nucleic Acids Res. 39, W339-W346. doi: 10.1093/nar/gkr466

Nemoto, T., Maruyama, J., and Kitamoto, K. (2012). Contribution ratios of amyA, amyB, amyC genes to high-level alpha-amylase expression in Aspergillus oryzae. Biosci. Biotechnol. Biochem. 76, 1477-1483. doi: 10.1271/bbb.120142

Nierman, W. C., Yu, J., Fedorova-Abrams, N. D., Losada, L., Cleveland, T. E., Bhatnagar, D., et al. (2015). Genome Sequence of Aspergillus flavus NRRL 3357, a strain that causes aflatoxin contamination of food and feed. Genome Announc. 3:e00168-15. doi: 10.1128/genomeA.00168-15

Priebe, S., Kreisel, C., Horn, F., Guthke, R., and Linde, J. (2015). FungiFun2: a comprehensive online resource for systematic analysis of gene lists from fungal species. Bioinformatics 31, 445-446. doi: 10.1093/bioinformatics/btu627

Purugganan, M. D., and Fuller, D. Q. (2009). The nature of selection during plant domestication. Nature 457, 843-848. doi: 10.1038/nature07895

Quinlan, A. R., and Hall, I. M. (2010). BEDTools: a flexible suite of utilities for comparing genomic features. Bioinformatics 26, 841-842. doi: 10.1093/ bioinformatics/btq033

Rajamani, S., and Hilda, A. (1987). Plate assay to screen fungi for proteoltyic activity. Curr. Sci. 56, 1179-1181.

Rinker, D. C., Specian, N. K., Zhao, S., and Gibbons, J. G. (2019). Polar bear evolution is marked by rapid changes in gene copy number in response to dietary shift. Proc. Natl. Acad. Sci. U.S.A. 116, 13446-13451. doi: 10.1073/pnas. 1901093116

Ropars, J., Didiot, E., Rodriguez de la Vega, R. C., Bennetot, B., Coton, M., Poirier, E., et al. (2020). Domestication of the emblematic white cheese-making fungus penicillium camemberti and its diversification into two varieties. Curr. Biol. 30, 4441-4453.e4. doi: 10.1016/j.cub.2020.08.082

Rubin, C. J., Zody, M. C., Eriksson, J., Meadows, J. R., Sherwood, E., Webster, M. T., et al. (2010). Whole-genome resequencing reveals loci under selection during chicken domestication. Nature 464, 587-591. doi: 10.1038/nature08832

Salque, M., Bogucki, P. I., Pyzel, J., Sobkowiak-Tabaka, I., Grygiel, R., Szmyt, M., et al. (2013). Earliest evidence for cheese making in the sixth millennium BC in northern Europe. Nature 493, 522-525. doi: 10.1038/nature11698
Simao, F. A., Waterhouse, R. M., Ioannidis, P., Kriventseva, E. V., and Zdobnov, E. M. (2015). BUSCO: assessing genome assembly and annotation completeness with single-copy orthologs. Bioinformatics 31, 3210-3212. doi: 10.1093/ bioinformatics/btv351

Stamatakis, A. (2014). RAxML version 8: a tool for phylogenetic analysis and post-analysis of large phylogenies. Bioinformatics 30, 1312-1313. doi: 10.1093/ bioinformatics/btu033

Stanke, M., and Waack, S. (2003). Gene prediction with a hidden Markov model and a new intron submodel. Bioinformatics 19 Suppl 2, ii215-ii225. doi: 10. 1093/bioinformatics/btg1080

Steensels, J., Gallone, B., Voordeckers, K., and Verstrepen, K. J. (2019). Domestication of industrial microbes. Curr. Biol. 29, R381-R393. doi: 10.1016/ j.cub.2019.04.025

Steenwyk, J. L., Mead, M. E., Knowles, S. L., Raja, H. A., Roberts, C. D., Bader, O., et al. (2020). Variation among biosynthetic gene clusters, secondary metabolite profiles, and cards of virulence across aspergillus species. Genetics 216, 481-497. doi: 10.1534/genetics.120.303549

Thammarongtham, C., Nookaew, I., Vorapreeda, T., Srisuk, T., Land, M. L., Jeennor, S., et al. (2018). Genome characterization of oleaginous aspergillus oryzae bcc7051: a potential fungal-based platform for lipid production. Curr. Microbiol. 75, 57-70. doi: 10.1007/s00284-017-1350-7

Tominaga, M., Lee, Y. H., Hayashi, R., Suzuki, Y., Yamada, O., Sakamoto, K., et al. (2006). Molecular analysis of an inactive aflatoxin biosynthesis gene cluster in Aspergillus oryzae RIB strains. Appl. Environ. Microb. 72, 484-490. doi: 10.1128/aem.72.1.484-490.2006

Veltri, D., Wight, M. M., and Crouch, J. A. (2016). Simple Synteny: a web-based tool for visualization of microsynteny across multiple species. Nucleic Acids Res. 44, W41-W45.

Watarai, N., Yamamoto, N., Sawada, K., and Yamada, T. (2019). Evolution of Aspergillus oryzae before and after domestication inferred by large-scale comparative genomic analysis. DNA Res. 26, 465-472. doi: 10.1093/dnares/ dsz024

Wickham, H. (2009). ggplot2: Elegant Graphics for Data Analysis. New York, NY: Springer-Verlag.

Yu, G. (2020). Using ggtree to visualize data on tree-like structures. Curr. Protoc. Bioinformatics 69:e96.

Zhao, S., and Gibbons, J. G. (2018). A population genomic characterization of copy number variation in the opportunistic fungal pathogen Aspergillus fumigatus. PLoS One 13:e0201611. doi: 10.1371/journal.pone.0201611

Zimin, A. V., Marcais, G., Puiu, D., Roberts, M., Salzberg, S. L., and Yorke, J. A. (2013). The MaSuRCA genome assembler. Bioinformatics 29, 2669-2677. doi: 10.1093/bioinformatics/btt476

Conflict of Interest: The authors declare that the research was conducted in the absence of any commercial or financial relationships that could be construed as a potential conflict of interest.

Copyright (C) 2021 Chacón-Vargas, McCarthy, Choi, Wang, Yu and Gibbons. This is an open-access article distributed under the terms of the Creative Commons Attribution License (CC BY). The use, distribution or reproduction in other forums is permitted, provided the original author(s) and the copyright owner(s) are credited and that the original publication in this journal is cited, in accordance with accepted academic practice. No use, distribution or reproduction is permitted which does not comply with these terms. 